\title{
Temperature-dependent of Nonlinear Optical Conductance of Graphene-based Systems in High-intensity Terahertz Field
}

\author{
Jing Lv ${ }^{1}$, Rui-yang Yuan ${ }^{2, *}$, Hui Yan ${ }^{1, *}$
}

(Received 11 November 2013; accepted 10 February 2014; published online 20 March 2014)

\begin{abstract}
For multi-photon processed with the linear dispersion in the high-intensity terahertz (THz) field, we have systematically investigated the temperature-dependent nonlinear optical response of graphene-based systems, including single layer graphene, graphene superlattice and gapped graphene. In the intrinsic single layer graphene system, it demonstrates that, at low temperature, nonlinear optical conductivities of the thirdand fifth-order are respectively five and ten orders of magnitude larger than the universal conductivity with high-intensity and low frequency $\mathrm{THz}$ wave.In the graphene superlattice and gapped graphene systems, the optical responses enhanced because of the anisotropic massless and massive Dirac fermions.
\end{abstract}

Keywords: Nonlinear; Graphene; Terahertz; Conductance; Transmittance

Citation: Jing Lv, Rui-yang Yuan and Hui Yan, "Temperature-dependent of Nonlinear Optical Conductance of Graphene-based Systems in High-intensity Terahertz Field", Nano-Micro Lett. 6(2), 153-162 (2014). http:// dx.doi.org/10.5101/nml.v6i2.p153-162

\section{Introduction}

Graphene is a single atomic layer of carbon atoms tightly packed into a highly symmetric two-dimensional honeycomb lattice [1]. The spectrum of graphene quasiparticles, electrons and holesare described by an effective Dirac equation with a vanishing effective mass [2]. The massless energy dispersion of graphene quasiparticles leads to many unique physical properties, such as the electron-hole symmetry and a half-integer quantum Hall effect [3-5], a finite conductivity of $4 \mathrm{e}^{2} / \mathrm{h}$ even when the carrier concentration tends to zero [3], the strong suppression of weak localization [6-8] and ultrahigh carrier mobility [1]. Therefore, graphene is considered as a promising material for electronic applications.

The optical properties of graphene have been studied both experimentally [9-17] and theoretically [18-30]. In the terahertz to the far-infrared (FIR) spectral regime, optical conductance of graphene-based systems attracts many researchers' interests due to the ongoing search on viable terahertz detectors and emitters. Graphene is traditionally a poor conductor in this part of the spectrum, with the universal conductivity $\sigma_{0}=e^{2} / 4 \hbar$ leading to absorb only $2.3 \%$ at normal incidence per graphene layer [11]. As discussed by Miknailov et al. $[21,22]$, they developed a quasi-classical kinetic theory to predict the stronger nonlinear electromagnetic response of graphene under electromagnetic radiation at different frequencies ranging from microwaves to infrared. These studies show that irradiation of graphene by an electromagnetic wave of frequency $\omega$ should lead to excitation of odd high-harmonics (i.e. $3 \omega, 5 \omega, 7 \omega, \cdots)$. This may make graphene as a simple and natural frequency multiplier and open up exciting opportunities for using graphene in terahertz electronics. Subsequently, a series of theoretical works were carried out to seek for the strong nonlinear terahertz

\footnotetext{
${ }^{1}$ Laboratory of Thin Film Materials, Beijing University of Technology, Beijing 100022, China

${ }^{2}$ Center of Theoretical Physics, Department of Physics, Capital Normal University, Beijing 100048, China

*Corresponding author. E-mail: yuanry@cnu.edu.cn
} 
responses of graphene-based systems, such as single layer graphene (SLG) [23-25], bilayer graphene (BLG) [26], graphene p-n junction [27], graphene nanoribbons (GNRs) [28], semihydrogenated graphene (SHG) [29] and Kronig-Penney type (KP) graphene superlattice [30]. As studied by Hendry et al. in 2010 [15], they first measured the coherent nonlinear optical response of single- and few-layer graphene using four-wave mixing. Their results demonstrate that graphene exhibits a very strong nonlinear optical response in the nearinfrared spectral region. The large optical nonlinearity originates from the interband electron transitions and is eight orders of magnitude larger than the nonlinearities observed for dielectric materials without such transitions. Recent experimental progress presented the results on studying the carrier dynamics in epitaxially grown graphene at higher photon energies from 10 to $250 \mathrm{meV}$ [16], as well as the nonlinear $\mathrm{THz}$ response of graphene excited at electric field strengths in the nonperturbative regime [17].

The theoretical progresses have been shown that the linear energy dispersion of quasiparticles, electrons and holes in graphene leads to the strongly nonlinear optical response of this system in the terahertz to the FIR regime [21]. As a consequence, graphene is a rather strong nonlinear material. Wright and $\mathrm{Xu}$ et al. [23,24] adopted a quantum-mechanical approach to study the linear, third-order and fifth-order nonlinear optical response of intrinsic SLG under an electric field of around $10^{3} \mathrm{~V} / \mathrm{cm}$, which indicates these relative nonlinear terms make graphene a potential applications for photonic and optoelectronic device. Zhou et al. [25] also treated the same topic with more rigorous perturbation theory. Yee Sin Ang et al. [28, 29] investigated the optical conductivity of SHG and KP graphene superlattic. It has been shown that SHG possesses a unique potential as a two-color nonlinear material and the total optical response of the graphene superlattice was enhanced due to the formation of anisotropic Dirac fermions in the terahertz frequency region. It was also revealed that the band structure isotropy is not a prerequisite for the strong optical nonlinearity in graphene.

Previously letters only studied the third- and fifthorder nonlinear optical response to each graphene system with frequency respectively, and did not mention the temperature factors when calculating the relationship between the transmittance and the frequency in the terahertz regime. The main purpose of this paper is to theoretically investigate the temperature-dependent nonlinear optical conductance of different graphenebased systems in high-intensity terahertz field based on the simplified model for multiphoton process with linear dispersion. Firstly, we investigated the strong nonlinear optical response of intrinsic single layer graphene systematically, and obtained relationship between trans- mittance versus temperature and intensity. Then, we studied the nonlinear optical response of KP graphene superlattice,considering the anisotropy massless Dirac fermions. Moreover, the strong optical nonlinearity existed in gapped graphene was revealed.

\section{Theory and discussion}

\section{Massless Dirac fermions}

We adopted the approach that couples the massless Dirac fermions to the time-dependent electric field quantum mechanically to calculate the nonlinear terms, both in high-order electrical field and in multiple frequencies. When a time-dependent external field $E(t)=$ $E_{0} e^{i \omega t}$ is along the $x$-axis, the low energy tight binding Hamiltonian of graphene-based systems can be written as,

$$
H=v_{F}\left(\begin{array}{cc}
0 & p_{-}+e A_{-} \\
p_{+}+e A_{+} & 0
\end{array}\right)
$$

where $P_{ \pm}=P_{x} \pm \lambda \mathrm{i} P_{y}, A_{ \pm}=A=\left(E_{0} / \mathrm{i} \omega\right) e^{i \omega t}$ and $v_{F} \approx c / 300=10^{6} \mathrm{~m} / \mathrm{s}$ is the Fermi velocity. $\lambda$ is an anisotropy parameter, which modifies the $y$-direction group velocity by $v_{y}=\lambda v_{F}$, and can be continuously tuned in the range of $0<\lambda \leq 1$. The massless Dirac fermions is perfectly isotropic in all directions in the SLG which is the isotropic case $\lambda=1$, but travels anisotropically with $v_{F}$ in the direction along the periodicity of the KP potential ( $x$-direction) and reduced velocity of $\lambda v_{F}$ in the direction perpendicular periodicity (y-direction) in the KP graphene superlattice. The time-dependent two-component wave function can be expanded in the basis set,

$$
\psi(\mathbf{p}, t)=\sum_{n=0}^{\infty}\left[\begin{array}{c}
\alpha_{n}(\mathbf{p}) \\
\beta_{n}(\mathbf{p})
\end{array}\right] e^{i(n \omega-\varepsilon / \hbar) t}
$$

where $\varepsilon=v_{F}|P|$, by substituting Eq. (1) and (2) into the Schrödinger equation $i \hbar(\partial \psi / \partial t)=H \psi$. Due to the orthonormal relation of $e^{i n \omega t}$, we can write the coupled recursion relations for the spinor components,

$$
\begin{aligned}
& \alpha_{n}=\frac{e E_{0} v_{F}}{i \omega \hbar} \times \frac{v_{F} p_{-} \alpha_{n-1}+\left(v_{F} p-n \hbar \omega\right) \beta_{n-1}}{n \omega\left(n \hbar \omega-2 v_{F} p\right)} \\
& \beta_{n}=\frac{e E_{0} v_{F}}{i \omega \hbar} \times \frac{v_{F} p_{+} \beta_{n-1}+\left(v_{F} p-n \hbar \omega\right) \alpha_{n-1}}{n \omega\left(n \hbar \omega-2 v_{F} p\right)}
\end{aligned}
$$

The recursion relation couples the $n$ photon processes to the $n-1$ photon processes. From the solutions to Eq. (3) we can calculate the $n$th order total current,

$$
J_{n}=\frac{1}{2 \pi^{2}} \int N(\varepsilon) p \mathrm{~d} p \mathrm{~d} \theta \operatorname{Re}\left(\sum_{n=0}^{\infty} \alpha_{i}^{*} \beta_{n-i}+\beta_{i}^{*} \alpha_{n-i}\right)
$$

where $N(\varepsilon)=n_{F}(-\varepsilon)-n_{F}(\varepsilon)=\tanh \left(\varepsilon / 2 k_{B} T\right)$ is the temperature factor. 
The linear $(n=1)$ optical conductivity of graphenebased systems, which is a single photon process where an electron absorbs a photon making a transition from the valence band to the conduction band is descripted as,

$$
\sigma_{1}=\sigma_{1}(\omega)=\frac{\sigma_{0}}{\lambda} N\left(\frac{\hbar \omega}{2}\right)
$$

Because of time-reversal symmetry, the second and fourth order solutions make no contributions to the total current.

The third-order nonlinear $(n=3)$ optical conductivity is made up of two components: (i) $\sigma_{3}(\omega)$, singlefrequency term which corresponds to the absorption of two photons and the simultaneous emission of one photon; and (ii) $\sigma_{3}(3 \omega)$, triple-frequency term which corresponds to the simultaneous absorption of three photons, is descripted as,

$$
\begin{aligned}
\sigma_{3} & =\sigma_{3}(\omega)+\sigma_{3}(3 \omega) \\
& =\frac{\sigma_{0}}{\lambda} \frac{2 e^{2} v_{F}^{2} I}{\varepsilon_{0} c \hbar^{2} \omega^{4}}\left[N_{31}(\omega) e^{i \omega t}+N_{33}(\omega) e^{i 3 \omega t}\right]
\end{aligned}
$$

where $I\left(\mathrm{~W} / \mathrm{cm}^{2}\right)$ is the intensity and $\varepsilon_{0}$ is vacuum permittivity. The temperature factor are given by $N_{33}(\omega)=13 / 48 N(\hbar \omega / 2)-2 / 3 N(\hbar \omega)+15 / 16 N(3 \hbar \omega / 2)$ and $N_{31}(\omega)=2 N(\hbar \omega)$.

The fifth-order nonlinear $(n=5)$ optical conductivity is made up of three components: (i) $\sigma_{5}(\omega)$, singlefrequency term which corresponds to the absorption of three photons and the simultaneous emission of two photons; (ii) $\sigma_{5}(3 \omega)$, triple-frequency term which corresponds to the absorption of four photons and the simultaneous emission of one photon; (iii) $\sigma_{5}(5 \omega)$, fivefrequency term which corresponds to simultaneous absorption of five photons, is descripted as

$$
\begin{aligned}
\sigma_{5}= & \sigma_{5}(\omega)+\sigma_{5}(3 \omega)+\sigma_{5}(5 \omega) \\
= & \frac{\sigma_{0}}{\lambda} \frac{4 e^{4} v_{F}^{4} I^{2}}{\varepsilon_{0}^{2} c^{2} \hbar^{4} \omega^{8}}\left[N_{51}(\omega) e^{i \omega t}+\right. \\
& \left.N_{53}(\omega) e^{i 3 \omega t}+N_{55}(\omega) e^{i 5 \omega t}\right]
\end{aligned}
$$

where

$$
\begin{aligned}
N_{51}(\omega)= & \frac{159}{256} N\left(\frac{3 \hbar \omega}{2}\right) \\
N_{53}(\omega)= & \frac{2}{15} N(\hbar \omega)-\frac{867}{15360} N\left(\frac{3 \hbar \omega}{2}\right)+\frac{16}{135} N(2 \hbar \omega) \\
N_{55}(\omega)= & \frac{413}{23040} N\left(\frac{\hbar \omega}{2}\right)-\frac{2048}{23040} N(\hbar \omega)+ \\
& \frac{6102}{23040} N\left(\frac{3 \hbar \omega}{2}\right)-\frac{8192}{23040} N(2 \hbar \omega)+ \\
& \frac{4925}{23040} N\left(\frac{5 \hbar \omega}{2}\right)
\end{aligned}
$$

Considering both the linear and nonlinear (the thirdand fifth-order) terms, the total interband optical conductivity can be described by the formula,

$$
\begin{aligned}
\sigma^{\text {inter }}(\omega)= & \sigma_{1}+\sigma_{3}+\sigma_{5} \\
= & \frac{\sigma_{0}}{\lambda}\left\{N\left(\frac{\hbar \omega}{2}\right)+\frac{2 e^{2} v_{F}^{2} I}{\varepsilon_{0} c \hbar^{2} \omega^{4}}\left[N_{31}(\omega) e^{i \omega t}+\right.\right. \\
& \left.N_{33}(\omega) e^{i 3 \omega t}\right]+\frac{4 e^{4} v_{F}^{4} I^{2}}{\varepsilon_{0}^{2} c^{2} \hbar^{4} \omega^{8}}\left[N_{51}(\omega) e^{i \omega t}+\right. \\
& \left.\left.N_{53}(\omega) e^{i 3 \omega t}+N_{55}(\omega) e^{i 5 \omega t}\right]\right\}
\end{aligned}
$$

For intrinsic single layer graphene $(\lambda=1)$, the temperature dependence of linear $\sigma_{1}$ and third-order nonlinear optical conductivities $\sigma_{3}(\omega)$ and $\sigma_{3}(3 \omega)$ in unit of $\sigma_{0}$ is plotted in Fig. 1(a) at a frequency of $1 \mathrm{THz}$ under the intensity of $53 \mathrm{~W} / \mathrm{cm}^{2}$. We can find that the two nonlinear terms of the third-order conductivity decrease with temperature. At low temperature, the nonlinear term $\sigma_{3}(3 \omega)$ less than the linear conductance $\sigma_{1}$, and then stays as the same as the $\sigma_{1}$ even at room temperature. And the $\sigma_{3}(\omega)$ is greater than the linear conductance in the whole temperature regime. But when the frequencies below $0.7 \mathrm{THz}$, all the two third-order nonlinear terms are larger than the linear conductance in the whole temperature regime, and decrease rapidly with temperature than at $1 \mathrm{THz}$.

Figure 1(b) shows the intensity dependence of the third-order nonlinear optical conductance. It is demonstrated that when the intensity below $100 \mathrm{~W} / \mathrm{cm}^{2}$, the nonlinear term is so tiny that can be neglected. The ratio of third-order nonlinear optical conductivity and $\sigma_{0}$ increases with the intensity and the growth rate gradually slowed down. In the same intensity, the ratio decreases with increasing frequency. For high intensity and low frequencies, the nonlinear terms dominate; on the contrary, the linear terms dominate. The nonlinear conductance can be around an order of magnitude larger than the linear conductance when the intensity is $4000 \mathrm{~W} / \mathrm{cm}^{2}$.

Figure 2(a) shows the temperature dependence of linear $\sigma_{1}$ and fifth-order nonlinear optical conductivities $\sigma_{5}(\omega), \sigma_{5}(3 \omega)$ and $\sigma_{5}(5 \omega)$ in unit of $\sigma_{0}$ at a frequency of $1 \mathrm{THz}$ under the intensity of $120 \mathrm{~W} / \mathrm{cm}^{2}$. We can find that the three nonlinear terms decrease with increased temperature, and the $\sigma_{5}(\omega)$ decreases rapidly than the other two terms. At the low temperatures, the nonlinear term $\sigma_{5}(3 \omega)$ is less than the linear conductance, and then stays as the same as the linear conductance even at room temperature. The $\sigma_{5}(\omega)$ is larger than the linear conductance in the whole temperature regime. But the $\sigma_{5}(5 \omega)$ is less than the linear conductance and about one order magnitude lower than the $\sigma_{5}(\omega)$ in the low temperature regime. However, when the frequencies below $0.7 \mathrm{THz}$, all fifth-order nonlinear terms are larger than the linear conductance in the whole 
(a)

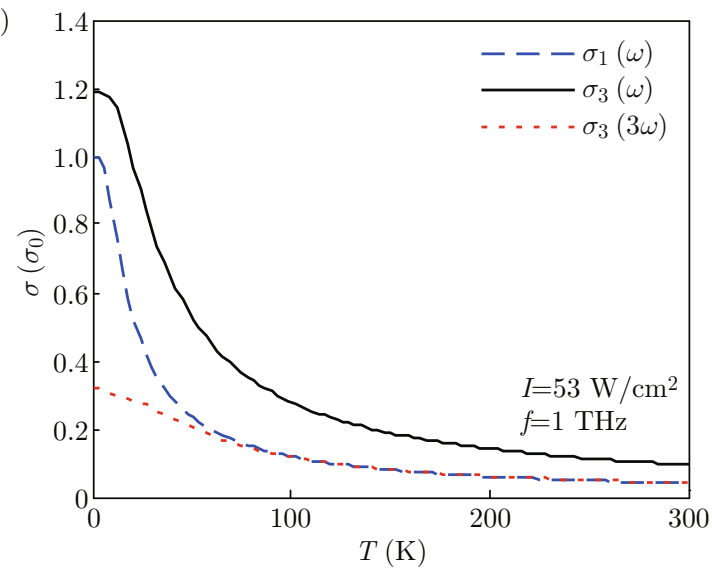

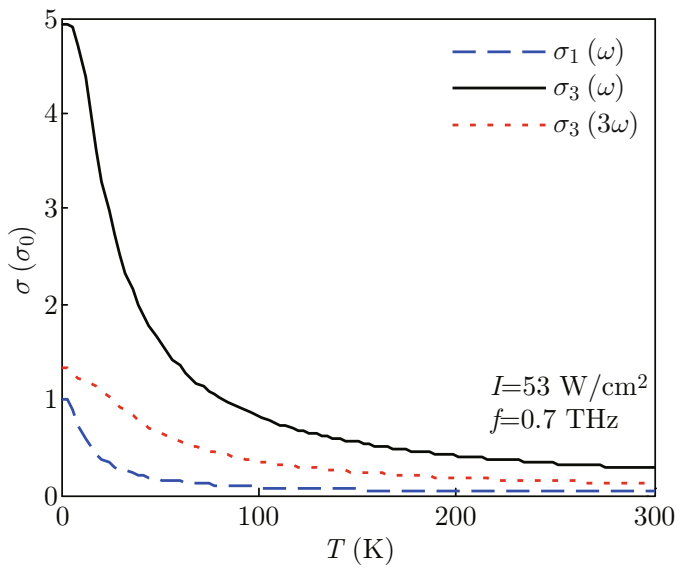

(b)

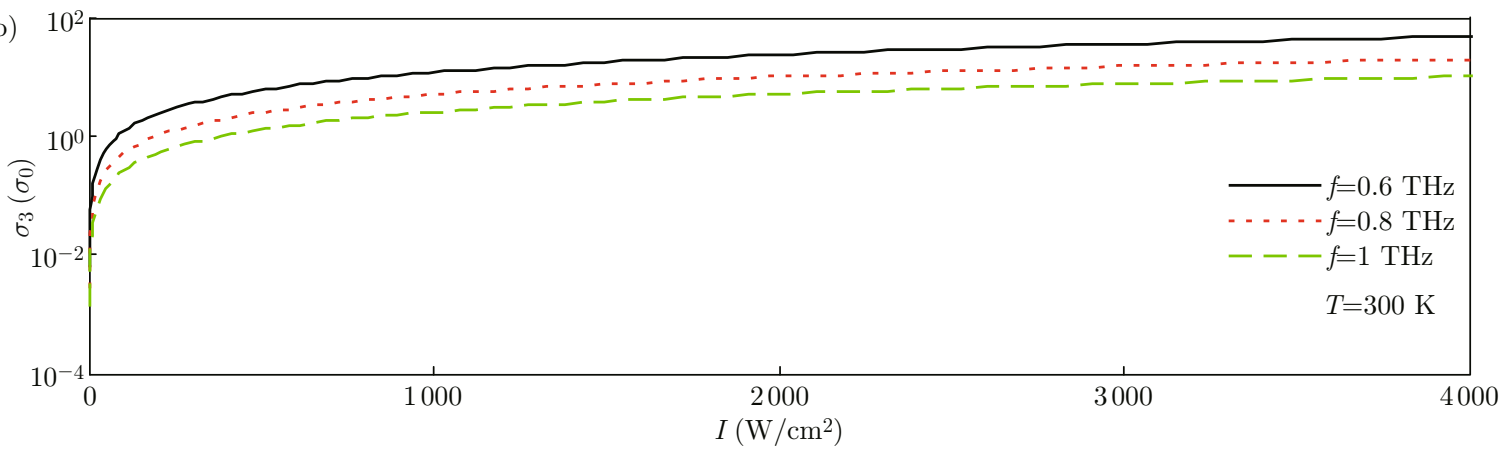

Fig. 1 For the SLG, (a) The linear and third-order nonlinear conductivity vs temperature for frequency of $1 \mathrm{THz}$ and 0.7 $\mathrm{THz}$; the intensity is $53 \mathrm{~W} / \mathrm{cm}^{2}$; (b) The third-order nonlinear conductivity vs intensity for frequency of $0.6,0.8,1 \mathrm{THz}$ at room temperature.

(a)

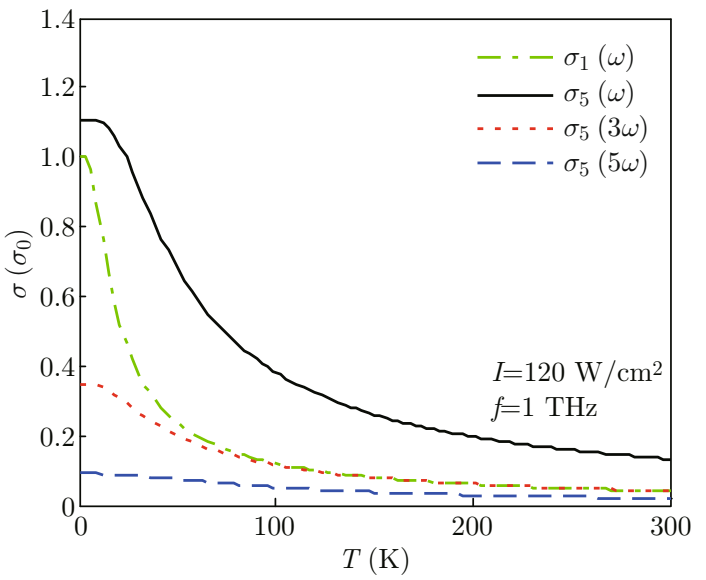

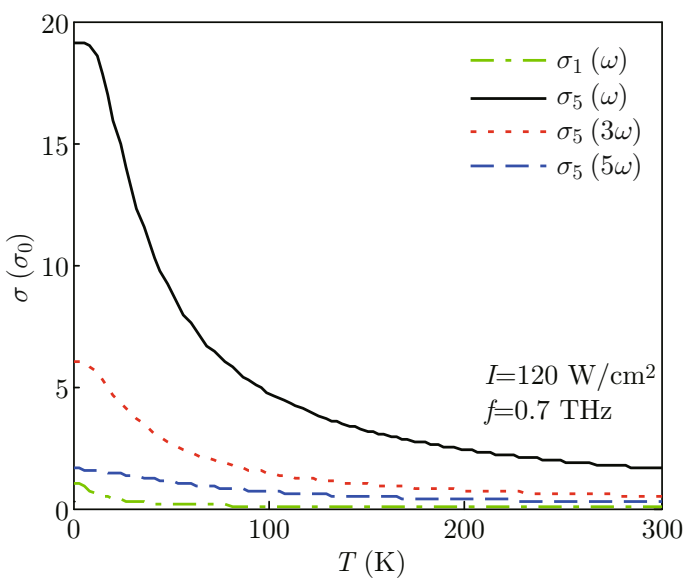

(b)

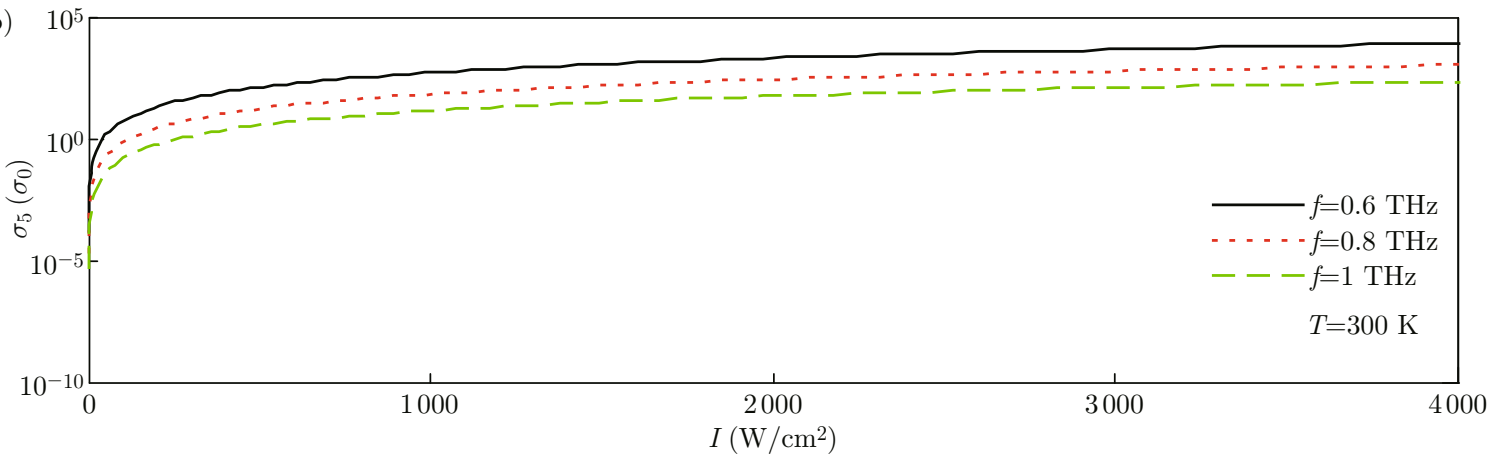

Fig. 2 For the SLG, (a) The linear and fifth-order nonlinear conductivity vs temperature for frequency of $1 \mathrm{THz}$ and 0.7 THz; the intensity is $120 \mathrm{~W} / \mathrm{cm}^{2}$; (b) The fifth-order nonlinear conductivity vs intensity for frequency of $0.6,0.8,1 \mathrm{THz}$ at room temperature. 
temperature regime, and decrease rapidly with increased temperature than those behave at $1 \mathrm{THz}$. Figure 2(b) illustrates the intensity dependence of the fifthorder nonlinear optical conductance. It is shown that when the intensity is weak, the nonlinear term is so tiny that can be neglected. The ratio of fifth-order nonlinear optical conductivity and $\sigma_{0}$ increases rapidly with the intensity which is faster than the third-order terms. Contrasting with Fig. 1(b) we can discover that the fifth-order nonlinear conductance can be about three orders magnitude larger than the third-order nonlinear conductance when the intensity is $4000 \mathrm{~W} / \mathrm{cm}^{2}$.

In general, we find that the nth order conductivity term is proportional to the parameter $Z=$ $\left(e v_{F} I^{0.5} / \hbar \omega^{2}\right)^{n-1}$. This means that at sufficiently high intensity and low frequency, nonlinear terms are relevant. To the present $\mathrm{THz}$ experiments, the value of intensity $1000 \mathrm{~W} / \mathrm{cm}^{2}$ is not so large for $\mathrm{THz}$ pulses, but it is very large for continuous wave (CW) sources. For example, if $\mathrm{CW} \mathrm{THz}$ laser generated $1 \mathrm{THz}$ with a spot diameter 300 micron, it would need around $1 \mathrm{~W}$ power to achieve this intensity. For lower frequency, it would require more power because the spot size should inevitably increase. However, with the development of $\mathrm{THz}$ technologies, this intensity value will eventually reach. Such a high-intensity $\mathrm{THz}$ wave yields nonnegligible numbers of photo-generated electrons and holes. In the absence of fast recombination processes, the photo-generated electrons and holes should increase the distribution functions and, thus, decrease the nonlinear conductivities. As a result, the actual nonlinear conductance is not so large above the intensity 1000 $\mathrm{W} / \mathrm{cm}^{2}$.

In order to contrast the third- and fifth-order nonlinear optical response more effectively, we plot the frequency dependent on the third- and fifth-order nonlinear optical conductance of SLG in unit of $\sigma_{0}$ for two different temperatures in Fig. 3. The intensity is 1000 $\mathrm{W} / \mathrm{cm}^{2}$. All nonlinear terms decrease rapidly with increased frequency. When frequencies below $1 \mathrm{THz}$, the nonlinear term dominates and the fifth-order nonlinear terms exceed the third-order nonlinear terms, which is about two orders of magnitude larger than that behaves in $0.5 \mathrm{THz}$. The huge nonlinearities in the frequency below $1 \mathrm{THz}$ are associated with the strong interaction of carriers with low-energy photons, which is characterized by the perturbation Hamiltonian inversely proportional to the photon energy. Within the frequency range $1.5 \sim 3 \mathrm{THz}$, the third-order nonlinear response is stronger than the fifth-order nonlinear response; however, they are so tiny that can be neglected completely. At the intensity around $1000 \mathrm{~W} / \mathrm{cm}^{2}$ and $f=1 \mathrm{THz}$, the value of $Z=10$ for third-order conductance and $Z=120$ for fifth-order conductance. However, for $f=3 \mathrm{THz}$, the resulting at about $Z=10^{-1}$ for third- order and $Z=10^{-2}$ for fifth-order nonlinear conductance, which is so small in the higher frequency that can be totally neglected.

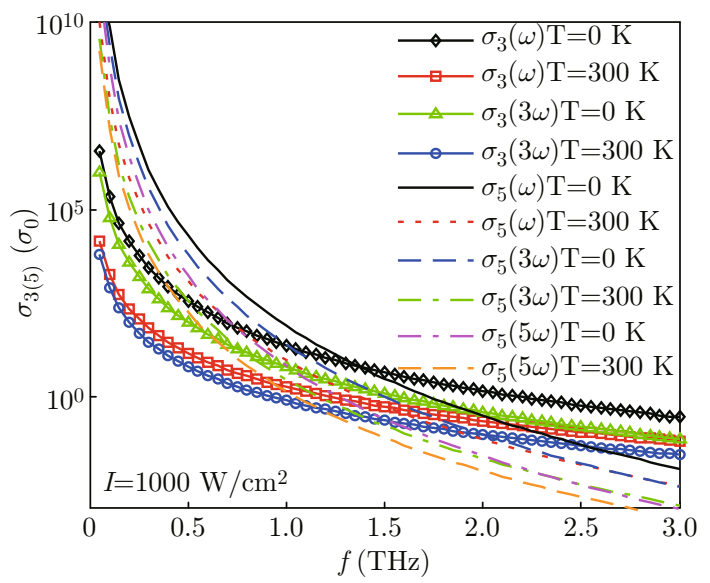

Fig. 3 For the SLG, the third-order $\left(\sigma_{3}(\omega)\right.$ and $\left.\sigma_{3}(3 \omega)\right)$ and fifth-order $\left(\sigma_{5}(\omega), \sigma_{5}(3 \omega)\right.$ and $\left.\sigma_{5}(5 \omega)\right)$ nonlinear conductivity vs frequency at zero and room temperatures; the intensity is $1000 \mathrm{~W} / \mathrm{cm}^{2}$.

In Fig. 4(a) we plotted the total interband optical conductivity of the frequency with $I=1000 \mathrm{~W} / \mathrm{cm}^{2}$ at $T=0 \mathrm{~K}$ and $300 \mathrm{~K}$, respectively. For frequencies below $0.5 \mathrm{THz}$, because the third- and fifth-order nonlinear terms play an important role compared with the linear term; the total interband optical conductivity is strongly decrease with frequency, about $10^{5} \Omega^{-1}$ at 0.1 $\mathrm{THz}$, which is more than five orders of magnitude larger than the universal conductivity. In the high-frequency part of the terahertz regime, the nonlinear terms play so small roles in the total interband optical conductivity that can be neglected completely. In the frequency range $3 \sim 10 \mathrm{THz}$, the total interband optical conductivity is almost unchanged with the frequency nearly equal to the universal conductivity $\sigma_{0} \approx 6.08 \times 10^{-5} \Omega^{-1}$.

We plotted the temperature dependent the total interband optical conductance with $I=1000 \mathrm{~W} / \mathrm{cm}^{2}$ for three different frequencies in Fig. 4(b). In the same frequency, the total interband optical conductance decreases with increasing temperature. The total interband optical conductance at $0 \mathrm{~K}$ is around two orders of magnitude higher than the total interband optical conductivity at $300 \mathrm{~K}$ for a frequency of $0.1 \mathrm{THz}$, and is about an order magnitude larger than that behaves at $1 \mathrm{THz}$. The large optical nonlinearity originates from the interband electron transitions and the temperaturedependence of the nonlinear conductivities is due to the decrease in the electron and hole distribution functions at integer multiples of half the photon energy. In the high-frequency part of the terahertz regime, the total interband optical conductivity is nearly equal to $\sigma_{0}$ at zero temperature and decreases slightly at room temperature. 


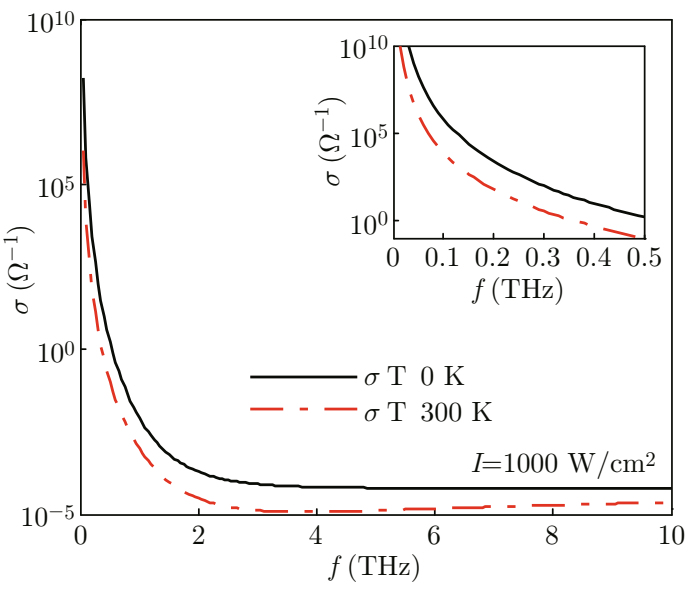

(a)

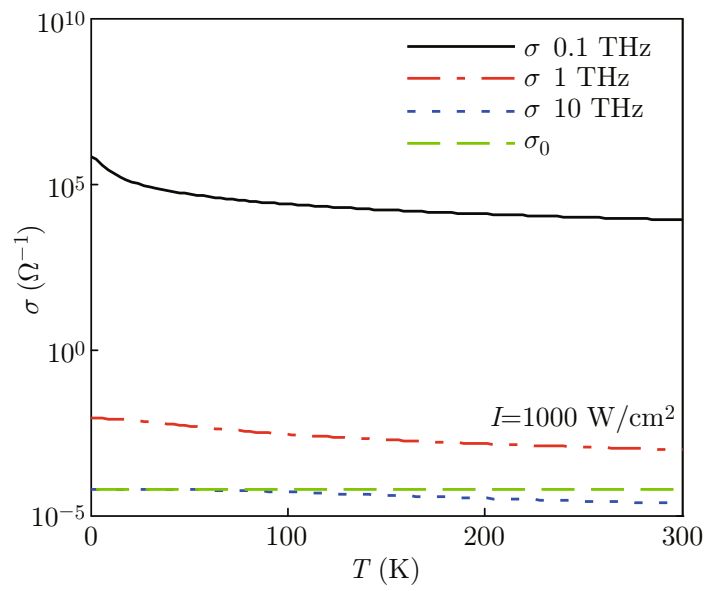

(b)

Fig. 4 (a) The total interband optical conductivity of SLG vs frequency at zero and room temperatures; the intensity is $1000 \mathrm{~W} / \mathrm{cm}^{2}$. The total optical conductivity in the low-frequency part of the terahertz regime (frequencies below $0.5 \mathrm{THz}$ ) is shown in the inset. (b) The total interband optical conductivity of SLG vs temperature for frequency of $0.1,1$ and 10 THz; the intensity is $1000 \mathrm{~W} / \mathrm{cm}^{2}$.

Optical transitions in graphene include interband and intraband transitions. Based on linearization of the tight binding Hamiltonian of graphene near the Dirac points of the first Brillouin zone, its total optical conductivity can be expressed as the sum of the two contributions,

$$
\sigma(\omega)=\sigma^{\text {inter }}(\omega)+\sigma^{\text {intra }}(\omega)
$$

The Drude model can estimate the intraband optical conductivity, which can be approximated as follows,

$$
\sigma^{\text {intra }}(\omega)=\frac{\mathrm{ie}^{2} E_{F}}{\pi \hbar^{2}\left(\omega+\mathrm{i} \tau^{-1}\right)}
$$

where $\tau$ is the relaxation time, the Fermi level $E_{F}$ is determined by the carrier concentration $n_{0}=$ $\left(E_{F} / \hbar v_{F}\right)^{2} / \pi$. The linear intraband conductivity associated with intraband transitions can be described as follows,

$$
\operatorname{Re}\left(\sigma_{1}^{\text {intra }}\right)=\frac{e^{2}}{2 \pi \hbar}\left(\frac{E_{F}}{\hbar}\right) \frac{\tau}{1+(\omega \tau)^{2}}
$$

At the intensity of $1000 \mathrm{~W} / \mathrm{cm}^{2}$, the generation rate of electrons and holes is about $10^{11} \mathrm{~cm}^{-2}$ in 1 ps. For the relaxation time of $1 \mathrm{ps}$, the real part of the linear intraband optical conductivity of SLG is around 0.13 $\Omega^{-1}$ at $0.1 \mathrm{THz}$ and $0.0044 \Omega^{-1}$ at $1 \mathrm{THz}$. Thus, the intraband conductivity is much weak than the interband conductivity that can be neglected completely.

According to the relation between the real part of optical conductivity and the transmittance [31], the transmittance of intrinsic SLG as follows,

$$
T_{r}=\frac{4 \varepsilon_{0}^{2}}{\left(2 \varepsilon_{0}+\sigma(\omega) / c\right)^{2}}
$$

In Fig. 5, considering the third- and fifth-order nonlinear responses and the temperature factor, we plot the transmittance versus the temperature for a frequency of $0.1,0.2,0.3$ and $1 \mathrm{THz}$ respectively with $I=1$ $\mathrm{W} / \mathrm{cm}^{2}$. At low frequencies $(0-0.2 \mathrm{THz})$, the transmittance is strongly decreased in the entire temperature regime by the nonlinear terms effect, in which the fifthorder terms play more important roles than the thirdorder terms. The transmittance decreases with decreasing temperature, and almost all absorbed at zero temperature for $0.1 \mathrm{THz}$. While the frequency is greater than $0.3 \mathrm{THz}$, both the third- and fifth-order nonlinear optical responses play so small roles in the total conductivity, consequently the transmittance is nearly equal to a constant slightly larger than $97.7 \%$ at room temperature.

Figure 6 illustrates the transmittance versus the intensity for a frequency of $0.1,0.5,1$ and $10 \mathrm{THz}$ respectively at room temperature. Considering the fifthorder nonlinear responses, the transmittance decreases quickly with increased intensity. When the intensity is around $10^{3} \mathrm{~W} / \mathrm{cm}^{2}$ and the frequency below $0.5 \mathrm{THz}$, the single layer intrinsic graphene is almost entirely absorption. However, in the high-frequency part of the terahertz regime, the transmittance is nearly equal to a constant slightly larger than $97.7 \%$, which is the same as the situation in the visible regime, and almost not changed by the increase of the intensity.

In the KP graphene superlattice $(0<\lambda<1)$, the group velocity of quasiparticle no longer travels with uniform $v_{F}$ in all directions. From the Eq. (6) and (7), the third- and fifth-order nonlinear optical conductances of graphene superlattice are all universally enhanced by a factor of $1 / \lambda$. In Fig. $7(\mathrm{a})$, the third-order nonlinear optical conductivities $\sigma_{3}(\omega)$ and $\sigma_{3}(3 \omega)$ at 

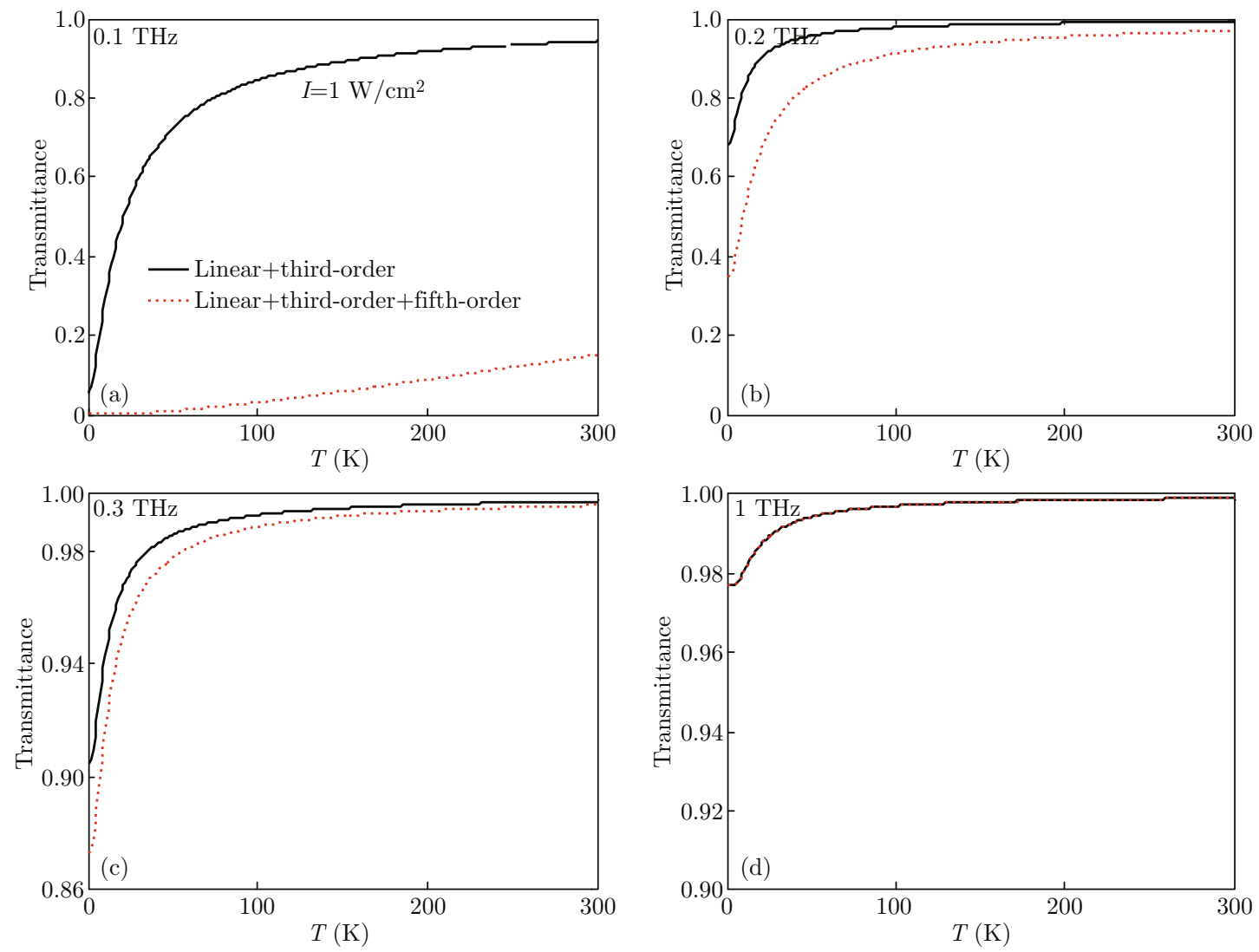

Fig. 5 The transmittance of intrinsic SLG vs temperature for frequency of $0.1,0.2,0.3$ and $1 \mathrm{THz}$; the intensity is $1 \mathrm{~W} / \mathrm{cm}^{2}$.
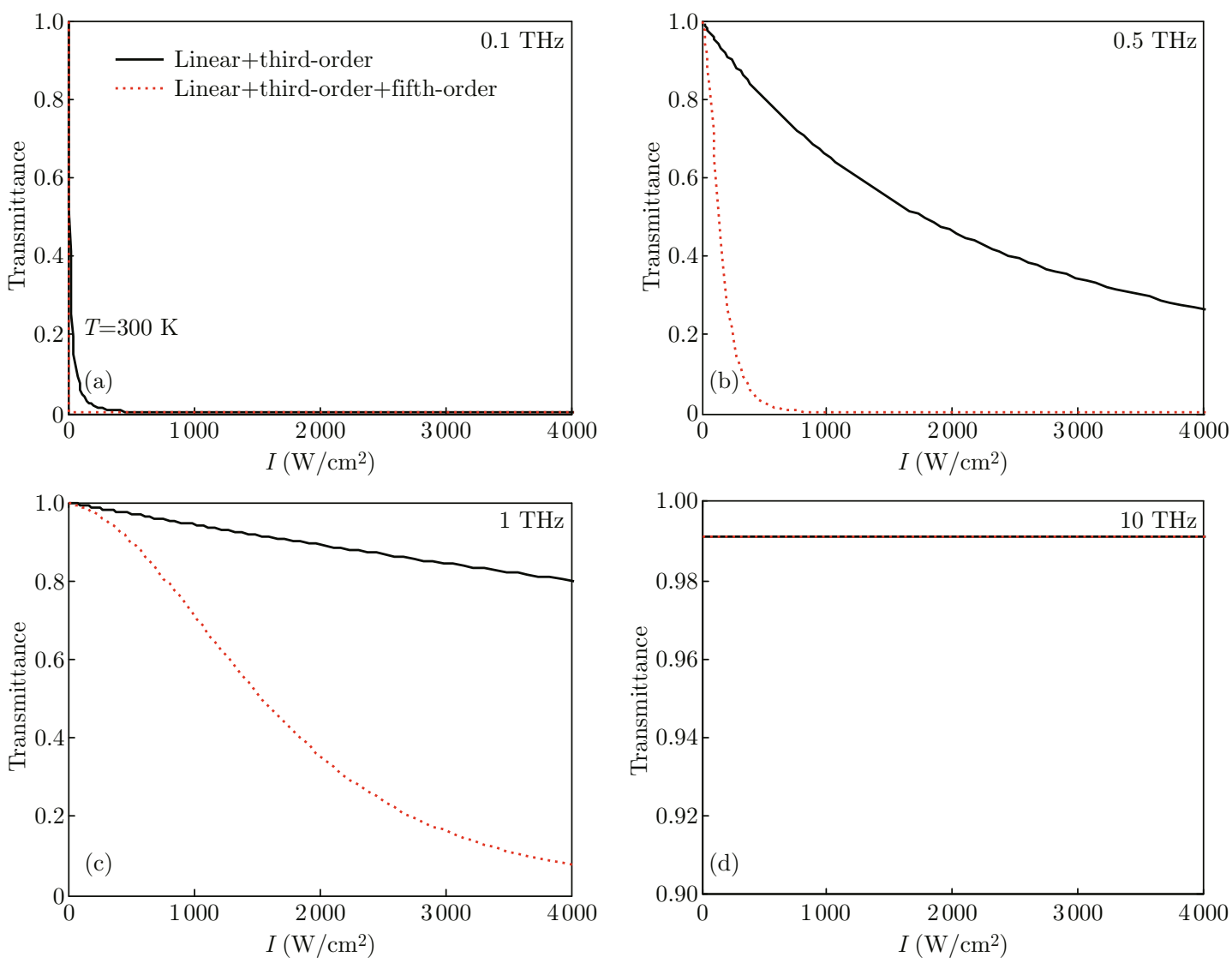

Fig. 6 The transmittance of intrinsic SLG vs electric field for frequency of $0.1,0.5,1$ and 10 THz at room temperature. 

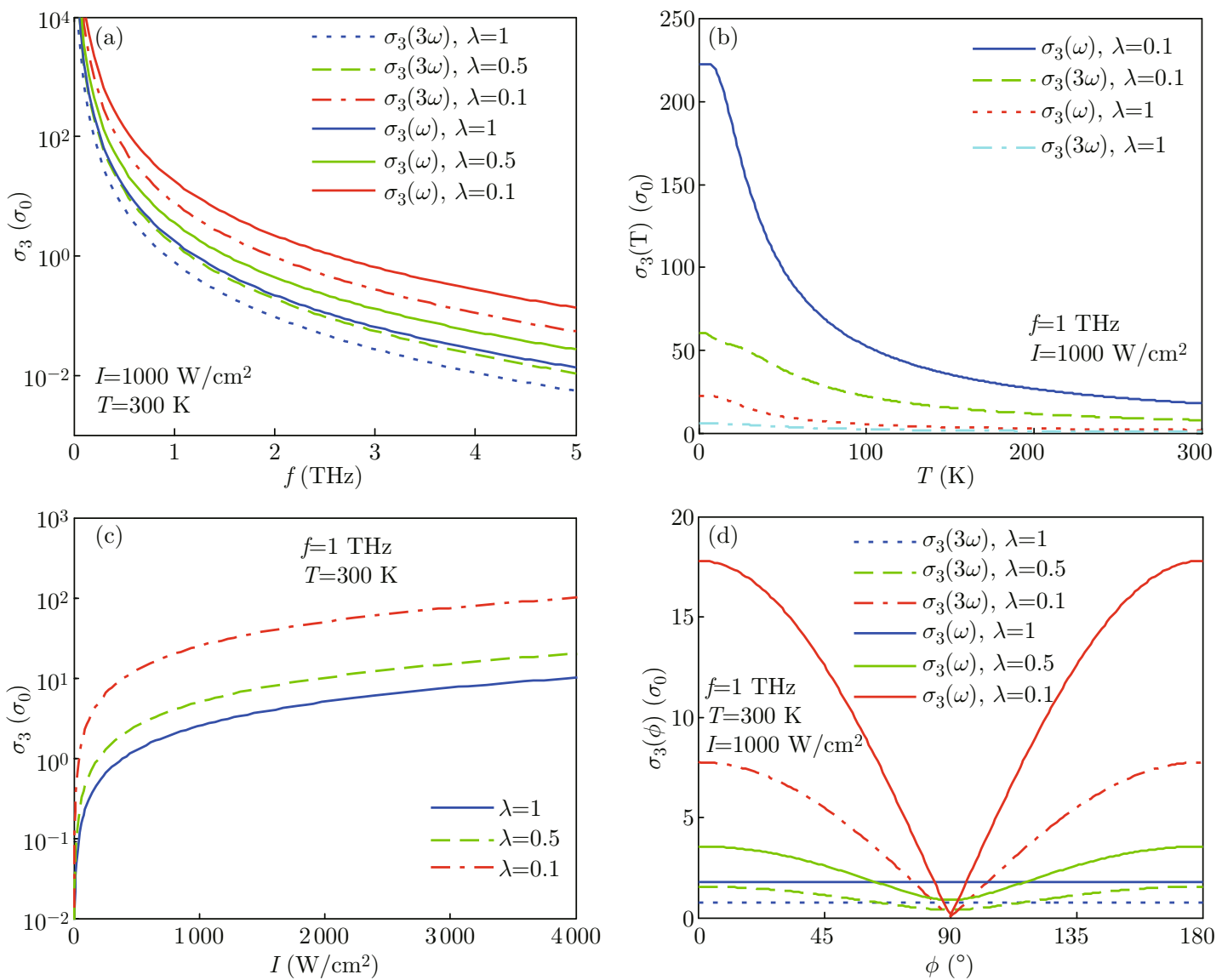

Fig. 7 For the SLG $(\lambda=1)$ and KP graphene superlattice $(0<\lambda<1)$, (a) The third-order nonlinear conductivity vs frequency for the intensity is $1000 \mathrm{~W} / \mathrm{cm}^{2}$ at room temperature; (b) The third-order nonlinear conductivity vs temperature for frequency of $1 \mathrm{THz}$; the intensity is $1000 \mathrm{~W} / \mathrm{cm}^{2}$; (c) The third-order nonlinear conductivity vs intensity for frequency at $1 \mathrm{THz} ; T=300 \mathrm{~K}$; (d) The third-order nonlinear conductivity vs angle $\phi$ for frequency of $1 \mathrm{THz}$ at room temperature; the intensity is $1000 \mathrm{~W} / \mathrm{cm}^{2}$.

$T=300 \mathrm{~K}$ and $I=1000 \mathrm{~W} / \mathrm{cm}^{2}$ are plotted for different $\lambda$. In comparison to the isotropic case (SLG), the nonlinear responses of graphene superlattice are universally enhanced by the anisotropy factor when the external field aligns with the supperlattice periodicity. The temperature dependence of the third-order nonlinear optical conductivitiesis plotted in Fig. 7(b) at $1 \mathrm{THz}$ and the intensity of $1000 \mathrm{~W} / \mathrm{cm}^{2}$. The two nonlinear terms decrease with increasing temperature, which is the same as the isotropic case but enhanced by a factor of $1 / \lambda$. Figure 7 (c) shows the intensity dependence of the third-order nonlinear optical conductance for different $\lambda$. For the KP graphene superlattice, the $y$-direction group velocity is reduced to $v_{y}=\lambda v_{F}$, the $y$-component momentum $p_{y}$ in an equi-energy slice becomes larger, so the overall larger momentum of the charge carrier is the reason for the enhancement of the optical conductance. In a more general case, when $E$ is in any angle $\phi$ as measured from the $x$-axis, the combine effect of the $x$-directional $\sigma(x)$ and $y$-directional $\sigma(y)$, the total angular dependence conductivity, measured along the $\phi$-direction, as follows, $\sigma(\phi)^{2}=\sigma(x)^{2}+\sigma(y)^{2}$, the ratio as follows, $\sigma_{n}(\phi, j \omega) / \sigma_{n}(j \omega)=\left(\cos ^{2}(\phi) / \lambda^{2}+\lambda^{2} \sin ^{2}(\phi)\right)^{1 / 2}$. The $\phi-$ dependence of the total third-order nonlinear conductivity is demonstrated in Fig. 7(d). The maximum conductance occurs at $\phi=0^{\circ}, 180^{\circ}$ when the $E$ is along the $x$-axis main enhanced by the factor of $1 / \lambda$. When the $\phi$ in the vicinity of $90^{\circ}, \sigma(y)$ becomes dominant and the influence of the factor $\lambda$ over the factor of $1 / \lambda$.

\section{Massive Dirac fermions}

The graphene with a finite band gap that could be created via breaking the symmetry of $\mathrm{A}$ and $\mathrm{B}$ sublattices in graphene-based system, the low-energy quasipaticles into massive Dirac fermions which are described by the finite-mass Dirac equation $H_{\Delta}=$ $\sigma_{x} p_{x}+\lambda \sigma_{y} p_{y}+(\Delta / 2) \sigma_{z}$. For the typical band gap value of $\Delta=0.03 \mathrm{eV}$, the linear and third-order nonlinear optical conductivities at low temperature are plotted in Fig. 8. It is shown that the gapped graphene also exists a strong nonlinear optical response by the three-photon 
process $\sigma_{3}(3 \omega)$ even when the energies lower than the band gap (sub-gap frequency regime) where the linear response is forbidden. And by changing anisotropy parameter, the nonlinear optical response is similarly tuned-up $1 / \lambda$. So this kind of gapped graphene as twocolor nonlinear material can be applied to nonlinear optics since the linear process is fully suppressed in the terahertz regime.

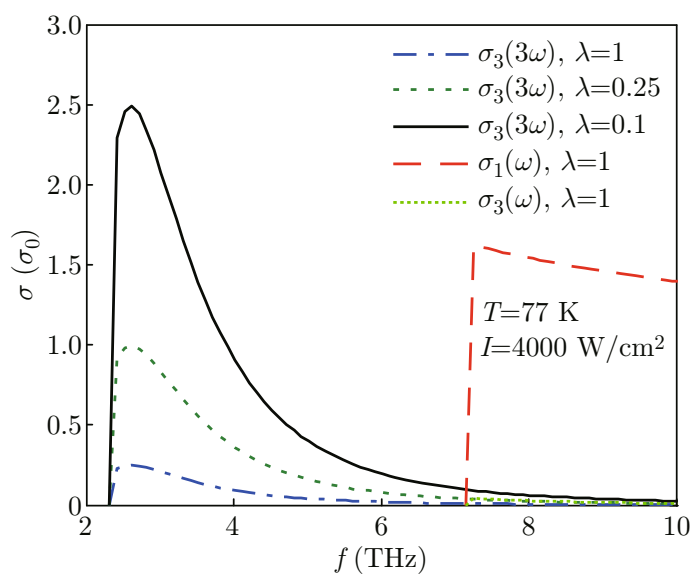

Fig. 8 The linear and third-order nonlinear optical conductivities of gapped graphene at low temperature $77 \mathrm{~K}$. The intensity is $1000 \mathrm{~W} / \mathrm{cm}^{2}$.

\section{Conclusion}

Depends on a theoretical investigation of the linear and nonlinear (the third- and fifth-order) optical conductivity, we have shown that graphene-based systems exhibit strong nonlinear optical response in the terahertz regime. Our results demonstrate that at low temperature, the third- and fifth-order nonlinear optical conductivities in the intrinsic single layer graphene system are more than five and ten orders of magnitude respectively, larger than the universal conductivity at the intensity around $1000 \mathrm{~W} / \mathrm{cm}^{2}$ and the frequency below $1 \mathrm{THz}$. In addition, the strong temperature-dependent nonlinear optical responses make the transmittance of SLG decrease rapidly and induce strong absorption. In the KP graphene superlattice system, the anisotropic Dirac fermions enhanced the optical response by a factor of $1 / \lambda$ and this kind of enhancement also occurs in gapped graphene system. These properties make graphene-based systems a potential candidate as a terahertz detector.

\section{Acknowledgement}

This work is supported by the National Special Fund for the Development of Major Research Equipment and Instruments (project No. 2011YQ13001805), and theFoundation of Beijing Educational Commission, China
(Grant No. KM201010028006).

\section{References}

[1] K. Geim, and K. S. Novoselov, "The rise of graphene", Nat. Mater. 6, 183-191 (2007). http://dx.doi.org/ $10.1038 /$ nmat 1849

[2] M. I. Katsnelson, "Graphene: carbon in two dimensions", Mater. Today10(1-2), 20-27 (2007). http:// dx.doi.org/10.1016/S1369-7021 (06) 71788-6

[3] K. S. Novoselov, A. K. Geim, S. V. Morozov, D. Jiang, M. I. Katsnelson, I. V. Grigorieva, S. V. Dubonos and A. A. Firsov, "Two-dimensional gas of massless Dirac fermions in graphene", Nature 438, 197-200 (2005). http://dx.doi.org/10.1038/nature04233

[4] Y. Zhang, Y. W. Tan, H. L. Stormer and P. Kim, "Experimental observation of the quantum Hall effect and Berry's phase in graphene", Nature 438, 201-204 (2005). http://dx.doi.org/10.1038/nature04235

[5] C. Berger, Z. Song, X. Li, X. Wu, N. Brown, C. Naud, D. Mayou, T. Li, J. Hass, A. N. Marchenkov, E. H. Konrad, P. N. First and W. A. de Heer, "Electronic confinement and coherence in patterned epitaxial graphene", Science 312(5777), 1191-1196 (2006). http://dx.doi.org/10.1126/science.1125925

[6] H. Suzuura and T. Ando, "Crossover from symplectic to orthogonal class in a two-dimensional honeycomb lattice", Phys. Rev. Lett. 89(26), 266603 (2002). http://dx.doi.org/10.1103/PhysRevLett. 89.266603

[7] S. V. Morozov, K. S. Novoselov, M. I. Katsnelson, F. Schedin, L. A. Ponomarenko, D. Jiang, and A. K. Geim, "Strong suppression of weak localization in graphene" , Phys. Rev. Lett. 97(01), 016801 (2006). http://dx.doi.org/10.1103/ PhysRevLett.97.016801

[8] D. V. Khveshchenko, "Electron localization properties in graphene", Phys. Rev. Lett. 97(03), 036802 (2006). http://dx.doi.org/10.1103/PhysRevLett. 97.036802

[9] R. S. Deacon, K.-C. Chuang, R. J. Nicholas, K. S. Novoselov and A. K. Geim, "Cyclotron resonance study of the electron and hole velocity in graphene monolayers", Phys. Rev. B 76(08), 081406(R) (2007). http://dx.doi.org/10.1103/PhysRevB.76.081406

[10] Z. Jiang, E. A. Henriksen, L. C. Tung, Y.-J. Wang, M. E. Schwartz, M. Y. Han, P. Kim and H. L. Stormer, "Infrared spectroscopy of landau levels of graphene", Phys. Rev. Lett. 98(19), 197403 (2007). http://dx. doi.org/10.1103/PhysRevLett.98.197403

[11] R. R. Nair, P. Blake, A. N. Grigorenko, K. S. Novoselov, T. J. Booth, T. Stauber, N. M. R. Peres and A. K. Geim, "Fine structure constant defines visual transparency of graphene", Science 320(5881), 13081308 (2008). http://dx.doi.org/10.1126/science. 1156965

[12] E. A. Henriksen, Z. Jiang, L. C. Tung, M. E. Schwartz, M. Takita, Y.-J. Wang, P. Kim and H. L. Stormer, 
"Cyclotron resonance in bilayer graphene", Phys. Rev. Lett. 100(08), 087403 (2008). http://dx.doi.org/10. 1103/PhysRevLett. 100.087403

[13] Z. Q. Li, E. A. Henriksen, Z. Jiang, Z. Hao, M. C. Martin, P. Kim, H. L. Stormer and D. N. Basov, "Dirac charge dynamics in graphene by infrared spectroscopy", Nature Physics4, 532-535 (2008). http:// dx.doi.org/10.1038/nphys989

[14] K. F. Mak, M. Y. Sfeir, Y. Wu, C. H. Lui, J. A. Misewich and T. F. Heinz, "Measurement of the optical conductivity of graphene", Phys. Rev. Lett. 101(19), 196405 (2008). http://dx.doi.org/10. 1103/PhysRevLett. 101.196405

[15] E. Hendry,P. J. Hale, J. Moger, A. K. Savchenko andS. A. Mikhailov, "Coherent nonlinear optical response of graphene", Phys. Rev. Lett. 105, 097401 (2010). http://dx.doi.org/10.1103/PhysRevLett. 105.097401

[16] S. Winnerl, M. Orlita, P. Plochocka, P. Kossacki, M. Potemski, T. Winzer, E. Malic, A. Knorr, M. Sprinkle, C. Berger, W. A. de Heer, H. Schneider and M. Helm, "Carrier relaxation in epitaxial graphene photoexcited near the dirac point", Phys. Rev. Lett. 107(23), 237401 (2011). http://dx.doi. org/10.1103/PhysRevLett.107.237401

[17] Michael Woerner, Wilhelm Kuehn, Pamela Bowlan, Klaus Reimann and Thomas Elsaesser, "Ultrafast two-dimensional terahertz spectroscopy of elementary excitations in solids", New J. Phys. 15, 025039 (2013). http://dx.doi.org/10.1088/1367-2630/15/ 2/025039

[18] V. P. Gusynin and G. Shand Srapov, "Transport of Dirac quasiparticles in graphene: Hall and optical conductivities", Phys. Rev. B73(24), 245411 (2006). http://dx.doi.org/10.1103/PhysRevB.73.245411

[19] V. P. Gusynin, S. G. Sharapov and J. P. Carbotte, "Magneto-optical conductivity in graphene", J. Phys. Condens. Matter 19, 026222 (2007). http://dx.doi. org/10.1088/0953-8984/19/2/026222

[20] L. A. Falkovsky and S. S. Pershoguba, "Optical farinfrared properties of a graphene monolayer and multilayer", Phys. Rev. B76(15), 153410 (2007). http:// dx.doi.org/10.1103/PhysRevB .76.153410

[21] S. A. Mikhailov, "Non-linear electromagnetic response of graphene", Europhys Lett. 79(02), 27002 (2007). http://dx.doi.org/10.1209/0295-5075/79/27002
[22] S. A. Mikhailov and K. Ziegler, "Nonlinear electromagnetic response of graphene: Frequency multiplication and the self-consistent-field effects", J. Phys.: Condens. Matter 20, 384204(2008). http://dx.doi.org/ 10.1088/0953-8984/20/38/384204

[23] A. R. Wright, X. G. Xu, J. C. Cao and C. Zhang, "Strong nonlinear optical response of graphene in the terahertz regime", Appl. Phys. Lett. 95(07), 072101 (2009). http://dx.doi.org/10.1063/ 1.3205115

[24] X. G. Xu and J. C. Cao, "Nonlinear response induced strong absorptance of graphene in the terahertz regime", Mod. Phys. Lett. B 24(21), 2243-2249 (2010). http://dx.doi.org/10.1142/S0217984910024626

[25] Y. Zhou and M. W. Wu, "optical response of graphene under intense terahertz fields", Phys. Rev. B 83(24), 245436 (2011). http://dx.doi.org/10.1103/ PhysRevB. 83.245436

[26] Yee Sin Ang, Shareef Sultan and C. Zhang, "Nonlinear optical spectrum of bilayer graphene in the terahertz regime", Appl. Phys. Lett. 97(24), 243110 (2010). http://dx.doi.org/10.1063/1.3527934

[27] X. G. Xu, S. Sultan, C. Zhang and J. C. Cao, "Nonlinear optical conductance in a graphene pn junction in the terahertz regime", Appl. Phys. Lett. 97(01), 011907 (2010). http://dx.doi.org/10.1063/ 1.3462972

[28] A. R. Wright, J. C. Cao and C. Zhang, "Enhanced optical conductivity of bilayer graphene nanoribbons in the terahertz regime", Phys. Rev. Lett. 103(20), 207401 (2009).http://dx.doi.org/10.1103/ PhysRevLett. 103.20740

[29] Yee Sin Ang and C. Zhang, "Subgap optical conductivity in semihydrogenated graphene", Appl. Phys. Lett. 98(04), 042107 (2011). http://dx.doi.org/10.1063/ 1. 3549201

[30] Yee Sin Ang and C Zhang, "Enhanced optical conductance in graphene superlattice due to anisotropic band dispersion", J. Phys. D: Appl. Phys. 45(39), 395303 (2012). http://dx.doi.org/10.1088/0022-3727/45/ $39 / 395303$

[31] N. M. R. Peres, "The electronic properties of graphene and its bilayer", Vacuum 83(10), 1248-1252 (2009). http://dx.doi.org/10.1016/j.vacuum.2009.03.018 